\title{
Coulisses
}

Revue de théâtre

1 | Printemps 1990

Varia

\section{Entretien avec Philippe Vialèles}

\section{Philippe Vialèles et Rédaction}

\section{OpenEdition}

\section{Journals}

Édition électronique

URL : http://journals.openedition.org/coulisses/1543

DOI : $10.4000 /$ coulisses. 1543

ISSN : 2546-9460

\section{Éditeur}

Presses universitaires de Franche-Comté

\section{Édition imprimée}

Date de publication : 1 avril 1990

Pagination : 19-25

ISSN : 1150-594X

\section{Référence électronique}

Philippe Vialèles et Rédaction, «Entretien avec Philippe Vialèles », Coulisses [En ligne], 1 | Printemps 1990, mis en ligne le 04 juillet 2017, consulté le 27 octobre 2019. URL : http:// journals.openedition.org/coulisses/1543; DOI : 10.4000/coulisses.1543

Ce document a été généré automatiquement le 27 octobre 2019

Coulisses 


\title{
Entretien avec Philippe Vialèles
}

\author{
Philippe Vialèles et Rédaction
}

1 Représenté au Nouveau Théâtre dans le cadre de la célébration du Bicentenaire, Le Captif, dont le personnage principal est Louis XVI, n'est pas une pièce de commande d'inspiration conjoncturelle. Elle se situe dans le prolongement d'un travail que $\mathrm{Ph}$. Vialèles mène depuis plus de cinq ans sur le théâtre symbolique, le théâtre du mythe et de la quête du Graal autour de la figure centrale du roi.

L'image royale parcourt le théâtre depuis les Grecs jusqu'à Ionesco, d'Edipe Roi au Roi se meurt ; mais, précise l'auteur, Le Captif n'est ni une pièce royaliste ni une pièce républicaine ; c'est avant tout du théâtre.

2 Captif, Louis XVI l'est bien avant sa fuite à Varennes. Installé aux Tuileries, il donne l'image publique d'un monarque qui gouverne alors que lui-même sait qu'il n'est qu'un roi fantoche.

Juste avant de partir pour Varennes, Louis XVI écrit une lettre où il dit être un fantôme de roi, une image. Il part pour sortir du mensonge : s'il est arrêté, alors la preuve sera faite aux yeux de tous qu'il était captif. Si vous arrêtez quelqu'un, c'est qu'il n'a pas le droit de sortir, qu'il est prisonnier.

Sur la scène, des cercles, des figures rondes représentent l'enfermement, la prison, symbolisent les roues du temps, le temps qui tourne, les anneaux du Styx, fleuve des Morts. A la fin de la pièce, le cercle se métamorphose pour devenir roue avec un cœur central. Le cœur, pour Ph. Vialèles, est celui d'un corps, le corps de l'humanité. L'auteur donne en effet une interprétation très personnelle, quasi mystique, du personnage royal qui, par compassion, accepte de vivre la souffrance du peuple.

Le drame royal, c'est la compassion, c'est-à-dire vivre ce que vit celui qui est en face. Le roi manifeste en lui toute la misère du peuple. Il est roi dans la mesure où il est le serviteur, où il va manifester en lui ce qui se passe dans le monde; si quelque chose est cassé dans le monde, alors lui aussi va être cassé : c'est ça le grand mystère royal.

Dans la pièce, le roi essaie de jouer le Roi avec un grand R. «Il ne s'identifie jamais au Roi, il l'incarne. » Incarner n'est pas s'identifier. On rejoint là le problème de l'acteur et du personnage. La mort de Louis XVI marque pour les gardiens la fin de leur fonction. «Gardiens nous ne sommes plus» disent-ils. Leur devenir fait partie du mystère 
d'après le spectacle. Une pièce se termine, une représentation s'achève et les acteurs disent : "notre rôle est terminé ». Que se passe-t-il après? "C'est là la beauté du théâtre ; on retourne à la nuit, la nuit du réel, à notre réel peut-être. » La fin du Captif est d'une grande ambiguïté tragique. «Les gardiens comprennent que le roi n'est pas séparé d'eux, qu'au contraire il fait partie d'eux-mêmes. " Les acteurs et le roi sont ensemble sur une pierre où ils vivent ensemble le drame de l'incarnation. « La tragédie est toujours ambiguë car les personnages vivent le même drame. » Pour Ph. Vialèles, c'est le discours politique qui, différenciant, classant les Bons et les Méchants, est séparateur.

5 Au théâtre, séparer les corps, les êtres n'est qu'un leurre : les acteurs jouent le même drame. «La séparation est une illusion ; la poésie relie. »

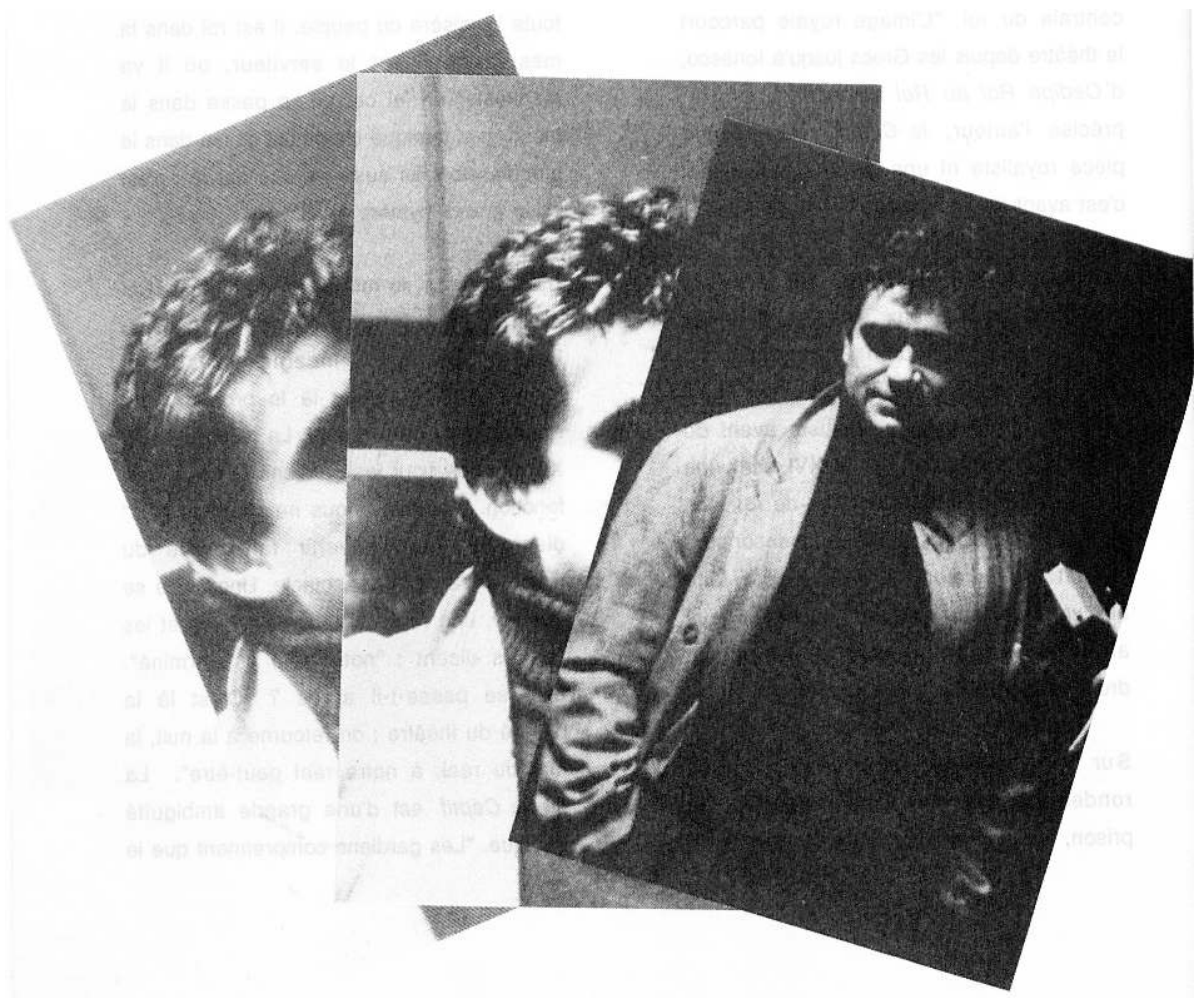

Philippe Vialèles.

(C) Valérie Goy.

\section{Après la pièce : impressions}

6 J'ai été agréablement surpris par rapport à ce que j'avais vu l'année dernière ; c'est beaucoup mieux.

7 C'est un spectacle très poignant, très poétique, avec un texte profond. Les comédiens sont bons. Par contre, j'ai trouvé le public exécrable ; il y avait comme une autocensure chez les gens qui se défendaient contre des émotions profondes : du rire à la place de l'émotion.

8 La première fois, j'ai été un peu déçu. J'ai pris le spectacle en pleine figure, très tortueux, difficile à comprendre mais en même temps posant des questions. Ce soir, deuxième lecture, les interrogations ont mûri. C'est un spectacle d'une grande beauté. 
9 Propos de l'auteur qui avoue ne pas très bien comprendre le qualificatif hermétique appliqué à sa pièce : "C'est hermétique parce qu'il est question de clef? de prison? Mais la clef qui ferme ouvre aussi.»

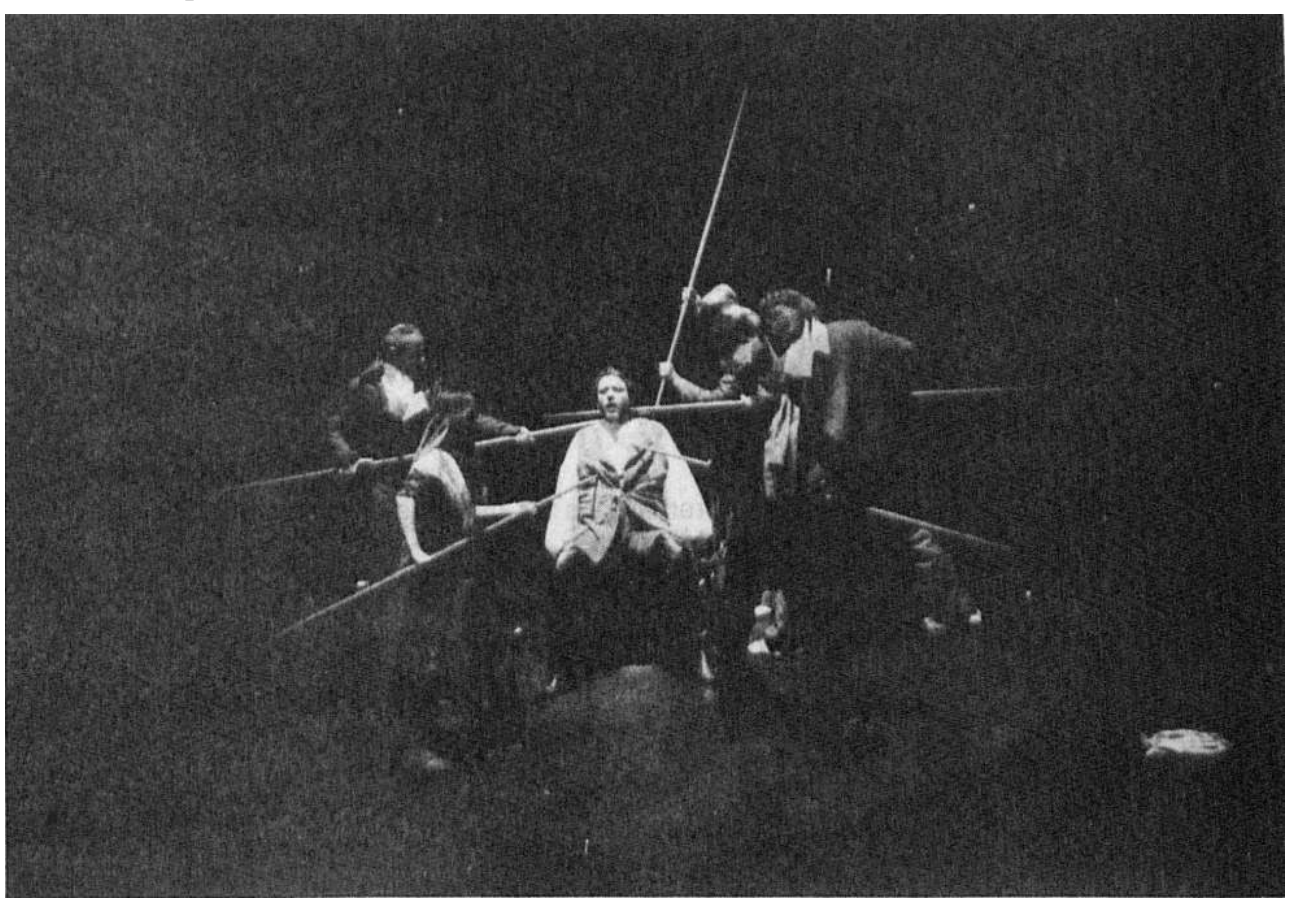

Le Captif.

(C) Valérie Goy.

\section{Le Captif de Philippe Vialèles (extraits inédits)}

\section{Acte II, scène 1}

\section{LOUIS}

O silence du monde, désastre immémorial, Obscurité qui me détruit, Nuit morcelée qui me sépare de ma tête! Et je n'ai plus de nom, plus de colère, Et j'attends dans le noir,

Et vous m'avez vaincu, jeté dans le silence,

Mais quelque chose en moi, mais quelque chose en moi vous répond! Un autre en moi, un roi en moi qui vous répond, Qui répond de vous !

\section{Acte IV, scène 6}

\section{LOUIS Recouvrant ses esprits}

À moi. La journée est finie.

Il faut partir.

Mes compagnons, mes petits frères,

C'est moi l'aîné,

C'est moi le père...

0 vous les morts,

Les grands et les petits,

Accourez, vous les morts!

Il frappe la pierre du pied 
Que mon pied vous réveille,

Qu'il vous fasse sortir,

Comme les rats de la cave !...

Corps démembré,

Tombé, brisé,

Que tous tes membres et tous tes os, sans en oublier un, jouent tous le même Drame,

Car il n'y en a qu'un!

Tambour. Il se lève et brandit son livre.

Les gardiens se réveillent.

JACQUES

Quand vient-il?

LOUIS

Maintenant !

GOST

Quand est-ce maintenant?

LOUIS

II faut attendre, il est ici !

JEANNE

Où es-tu?

GOST

Ici, c'est moi !

MARTHE

C'est moi !

JACQUES

C'est moi !

JEAN

C'est moi !

LOUIS

Ici, c'est Nous! Je suis l'Unique et je suis Tous !

GOST

Nous?

LOUIS

Nous ne sommes que des traces,

Des empreintes sur le sable,

Les lettres du mot obscur,

Reliées l'une à l'autre comme les Six Journées de la

Semaine Interminable,

Et il manque la dernière !...

Tambour

Mais l'angoisse nous frappe,

Elle brise le vase,

Et nous sommes comme l'eau répandue,

Et mes os se disloquent,

Et mon cœur, mon cœur en moi se fond comme la cire !

Je brûle ! J'ai soif !

Ma langue est sèche comme un tesson d'argile !...

Corps démembré

Sur l'enclume de la terre

Martelé par le Jour !

Terre craquelée, chair dévastée

Que surveillent les oiseaux, là-haut !...

Tambour

Voici le dernier coup,

Le plus brûlant, 
Un grand coup dans mon cœur !

0 blessure,

Vide,

Trace de l'Esprit!

Car il n'est rien que cette absence,

Que ce vent dans mon cœur,

Cette fenêtre par où passe le Vent!

Grand trou,

Où se plante l'Axe du Monde !

Je le sens,

Là en moi,

Qui se penche,

Et le char est remis sur ses roues !...

\section{Tambour}

Je suis l'Anneau d'alliance,

Je renoue le dedans au dehors,

L'Invisible au Visible,

Et la Rose s'épanouit !

Je suis le Grand Passage,

La Traversée de la Mer Rouge, le Flux et le Reflux !

Je reçois et je donne,

Et je suis le Moulin,

Et je relie les Ailes

À la Roue de la Terre,

Changeant l'épi rugueux en farine légère,

L'innombrable farine qui tourbillonne et blanchit le meunier!

Je suis le Moyeu rayonnant,

Et vous êtes les rayons, et vous êtes infinis, et jaillissants dans tous les sens !

Égalité !

Tous égaux dans mon cœur parce que tous infinis !

\section{Tambour}

Mon cœur est vide, mon cœur est lourd,

Et il tombe et il roule,

Mon corps est vide comme un tambour!

Tam-Tam,

Conque de peau tendue,

O médiateur, transformateur,

Frappé par les doigts du Batteur, là-haut,

Et tu résonnes en bas!

Mon corps est lourd, mon corps frappé !

Palombe foudroyée, je tombe comme un plomb, Mais un fil me retient, Je mesure l'abîme.

Tambour, Jean sort

Tendu,

Ecartelé,

Ma tête tu t'en vas,

Ma tête détachée

Par les doigts délicieux des divines musiciennes,

Et tu flottes sur la mer

Et tu poursuis ton chant !...

Et tu danses sur la houle

Tu roules dans ton panier !...

Berceau, barque funèbre,

Emporte le soleil,

Le roi qui meurt, emporte-le, 
Barque funèbre, radeau funèbre,

Embrasement sur la mer !...

Tu t'éloignes, tu t'éloignes,

O ma tête séparée,

Et les femmes sur la rive te regardent et agitent leurs mains,

Et tu chantes sur la mer :

"Je ne suis qu'une bouche

Qui expire l'Esprit,

Le souffle silencieux plus fort que la parole !

La Bouche,

L'Orifice,

Le Gouffre d'où surgissent les vents... »

Louis se tait et il s'immobilise, les yeux fermés, la tête penchée sur son épaule.

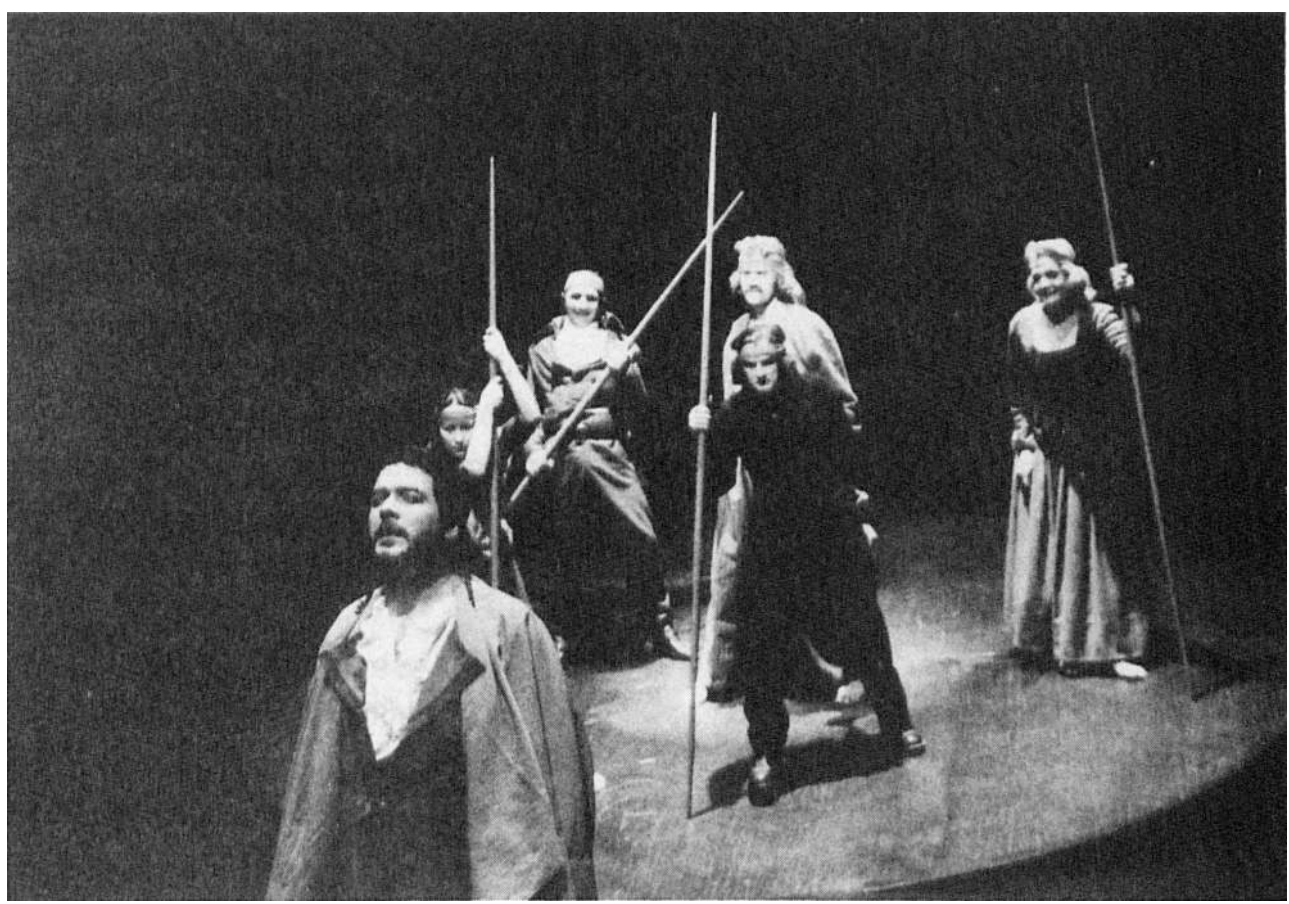

Le Captif.

(C) Valérie Goy. 